\title{
Development of a diagnostic technique for civil structures based on the model update of dynamic parameters
}

\author{
Francesco Ripamonti $^{\mathrm{a}}$, Alberto Bussini ${ }^{\mathrm{a}, \mathrm{b}}$, Ferruccio Resta $^{\mathrm{a}}$, Matias Ignacio Cortes Camus ${ }^{\mathrm{b}}$ \\ ${ }^{a}$ Politecnico di Milano, Department of Mechanics, Via La Masa 1, 20156 Milan (Italy)

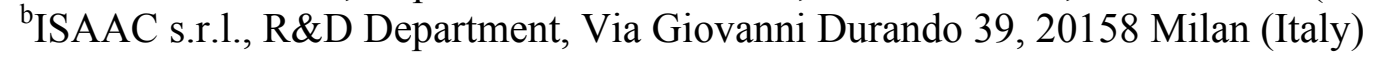

\begin{abstract}
Monitoring and diagnosis of civil structures has become a popular topic in the last years, especially through the use of non-invasive tests and techniques. Moreover, the spread of operational modal analysis, that exploit ambient excitations of structures to estimate their modal parameters, allowed to reduce the costs associated to the dynamic identification.

In this paper, a technique for the diagnosis of common civil structures, such as residential buildings or warehouses, is presented in order to identify local stiffness decreases that can potentially be associated to structural failures. The technique has been tested on a real warehouse built in 1960 and the results have been analyzed demonstrating the effectiveness of the methodology.
\end{abstract}

Keywords: Structural Health Monitoring, Operational Modal Analysis, Continuous Monitoring

\section{INTRODUCTION}

In this paper the structural conditions of a warehouse built in 1960 have been analyzed. A reliable Finite Element Model (FEM) of the building has been created to correctly analyze the presence of local damages in the structure. This has been performed by adopting the so-called FEM updating technique that belongs to the class of inverse problems in classical mechanics [1]. In the last years this technique attracted the interest of both research centers and industrial companies and it has been applied in the civil engineering field. It does not involve destructive tests on the structure resulting to be more rapid and less invasive. Moreover, the spread of Operational Modal Analysis (OMA), that exploits ambient excitations of structures to estimate their modal parameters, allowed to reduce the costs associated to the dynamic identification that usually implied to rent or buy heavy duty machines, such as vibrodines, to excite the motion of the structure in the interested frequency range. Indeed, this methodology requires the minimization of the residues computed from the differences between the modal parameters of the FEM and the ones identified thanks to the analysis of the structure vibration.

In the scientific literature it is possible to find different applications of this technique. For instance, in [2] an historic masonry tower has been studied: at first a dynamic identification of the building, using OMA algorithms implemented in the software ARTeMIS, and then the update of the FEM material mechanical properties, discretizing along the height of the tower, are carried out. Another example can be found in [3], where the data recorded on a 15-story steel frame building during the 2004 Parkfield earthquake have been used to analyze the dynamic properties of the building and update the FEM of the structure by minimizing the difference between the modal parameters (e.g. eigenfrequencies, mode shapes,...).

In this paper the general methodology is presented and finally applied on a real case, firstly demonstrating the reliability of the method by inserting artificial numerical damages that will be identified by the algorithms and secondly by applying the same procedure with the data recorded in an experimental campaign. This is a part of a framework developed in the last years in collaboration with ISAAC s.r.l., aiming at studying innovative solutions for the active vibration control [4-9] of civil structures during seismic events. 


\section{THE METHODOLOGY AND ITS IMPLEMENTATION}

As previously introduced, the model updating technique mainly consist in defining a residual vector containing the error between the modal parameters of the FEM and the identified ones from the modal analysis of the data collected from the real structure. At each optimization step, the model parameters that need to be optimized are adjusted and the objective function is evaluated allowing to understand the shape of the optimization function and to guess the values of the parameters to be used in the next iteration. However, being an inverse problem, it implies that infinite solutions are possible. For instance, each floor of a structure can be seen as made of springs with different stiffness in parallel, meaning that the equivalent stiffness related to the inter-story drift is the sum of all those values. This justifies the difficulty to isolate the local stiffness decrease of a single element such as a column. For this reason, it is important to define a suitable objective function limiting the risks related to local minima. For the aim of this work, not all the different types of optimizations and residual definitions will be presented but, for example, in [10] some of them are presented and applied to the model update of mechanical systems and in [11] other techniques specifically developed for the Civil engineering sector are described.

The main idea here proposed is that modal parameters can be distinguished between global and local information: natural frequencies are global while mode shapes are mostly related to a local information, even though these two characteristics are linked to each other.

To clarify this concept let's consider to perform the FEM update of a simple structure such as the one illustrated in Figure 1 and to define as main parameters to be adjusted the value of the elastic modulus of the concrete and a synthetic parameter for each column ranging between 0 and 1 , respectively representing the completely undamaged (0) and completely damaged case (1).

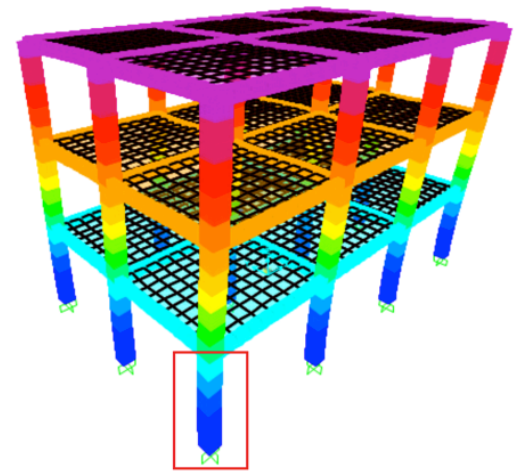

Figure 1 Example of FEM of a RC frame building

The damaged condition of a structural element can be associated to a local stiffness decrease. As an example, a concrete crack can be considered as a local decrease in the column section, thus a decrease in the moment of inertia and consequently in the total stiffness of the element, given by the multiplication of the elastic modulus and this value. In Table 1 the stiffness decrease due to the presence of cracks in the columns is summarized: in red the amount of cracked section is highlighted, thanks to which the related total decrease in the moment of inertia and thus the total stiffness decrease have been computed.

\begin{tabular}{|c|c|c|c|c|c|c|c|c|c|c|c|}
\hline $\begin{array}{c}\text { Percentage of } \\
\text { damage }\end{array}$ & $\mathbf{1 0 \%}$ & $\mathbf{2 0 \%}$ & $\mathbf{3 0 \%}$ & $\mathbf{4 0 \%}$ & $\mathbf{5 0 \%}$ & $\mathbf{6 0 \%}$ & $\mathbf{7 0 \%}$ & $\mathbf{8 0} \%$ & $\mathbf{9 0 \%}$ & $\mathbf{9 5 \%}$ & $\mathbf{9 9 \%}$ \\
\hline $\begin{array}{c}\text { Percentage } \\
\text { residual stiffness }\end{array}$ & $90 \%$ & $80 \%$ & $70 \%$ & $60 \%$ & $50 \%$ & $40 \%$ & $30 \%$ & $20 \%$ & $10 \%$ & $5 \%$ & $1 \%$ \\
\hline $\begin{array}{c}\text { Inertia moment } \\
\text { residual coeff. I22 }\end{array}$ & 0,95 & 0,89 & 0,84 & 0,77 & 0,71 & 0,63 & 0,55 & 0,45 & 0,32 & 0,22 & 0,1 \\
\hline $\begin{array}{c}\text { Inertia moment } \\
\text { residual coeff. I22 }\end{array}$ & 0,95 & 0,89 & 0,84 & 0,77 & 0,71 & 0,63 & 0,55 & 0,45 & 0,32 & 0,22 & 0,1 \\
\hline
\end{tabular}




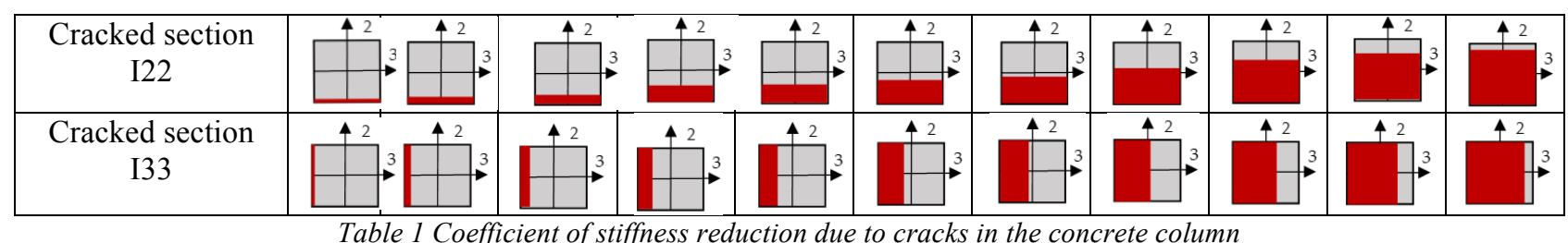

Table 1 Coefficient of stiffness reduction due to cracks in the concrete column

Now, by changing iteratively, the parameter related to the elastic modulus it will be noticed that all the natural frequencies of the system will decrease with a square root trend, but the mode shapes will always maintain the same shape. On the other hand, by changing the damage parameter of a group of columns of the structure belonging to the same region (e.g. the column selected in Figure 1), it will be noticed that the natural frequencies related to mode shapes that have mainly anti-nodes in that region, will decrease and also the related mode shapes will slightly change. So this can give an idea regarding how the structure parameters could influence the dynamics.

Thanks to these observations and different tests a suitable objective function and optimization procedure has been proposed following these steps:

1. A FEM of the building is created;

2. Vibrations of the building due to ambient excitation are recorded;

3. Suitable OMA methods are applied on the recorded data to guarantee reliable and robust identification of the real modal parameters of the structure;

4. The Modal Assurance Criterion (MAC) is computed between the analytical mode shapes obtained from the FEM and the ones obtained in the experiments, allowing to define their correspondences;

5. The weighted residue vectors are obtained from the normalized difference of the square roots of the natural frequencies $\left(r_{f}\right)$ and the differences between the square roots of the mode shapes $\left(r_{m}\right)$ :

$$
\begin{aligned}
& r_{f}=\frac{\left(\omega_{e}^{2}-\omega_{a}^{2}\right)}{\omega_{e}^{2}} \\
& r_{m}=\left[\psi_{e}^{2}-\psi_{a}^{2}\right]
\end{aligned}
$$

6. The error of the objective function $(\varepsilon)$ to be minimized w.r.t. the model parameters, is computed as the sum of the square roots of the residues at the previous step and opportunely weighted:

$\varepsilon=W \cdot r_{f}^{T} \cdot r_{f}+r_{m}^{T} \cdot r_{m}$

7. A first optimization loop changes iteratively the parameters associated to the materials $\left(x_{m}\right.$, such as the elastic modulus or density) weighting these values in order to change in a smaller range the variables that are most likely to be known from the beginning;

8. The main loop is run and the weight of the objective function changed in order to stress the importance of the mode shapes deformations due to the introduction of local stiffness decrease $\left(x_{i}\right)$ into the model to minimize the residues.

Going back to the example of Figure 1, the optimization procedure is performed by changing at each step the material and damage parameters and the related FEM analysis run to compute the analytical value of the modal properties and the objective function. This has been achieved thanks to a co-simulations on SAP2000, for the FEM analysis, and Matlab, for the optimization procedure.

In Figure 2 the objective functions obtained by changing linearly 2 parameters while computing the residues and the related error are presented. It can be clearly seen the effects previously introduced. In Figure 2(a) as the parameter $x_{m}$ changes, the error associated to the natural frequencies $\left(r_{f}\right)$ is highly affected while it is not sensitive to the parameter $x_{1}$ (damage of the first column). Similarly, from Figure 2(b) the frequency cost function seems not affected by a variation of the damage parameters $\left(x_{1}, x_{2}\right)$. 


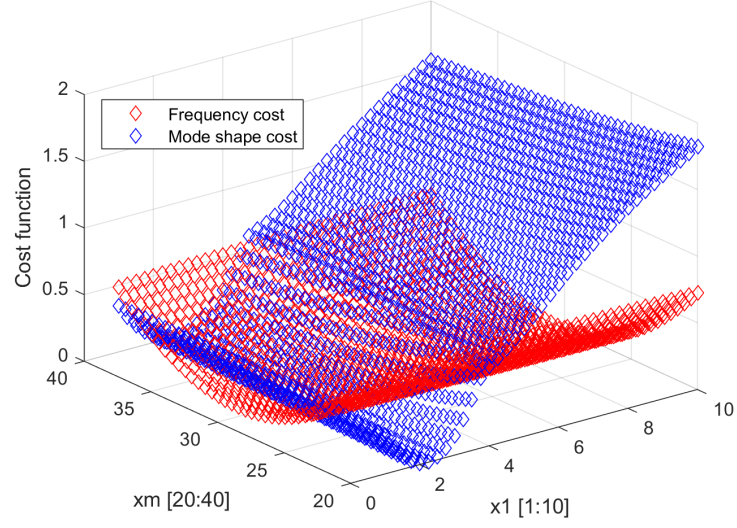

(a)

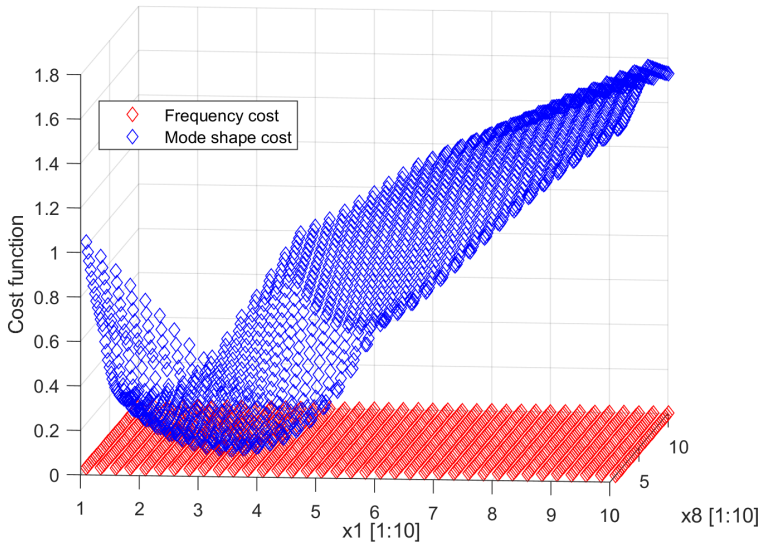

(b)

Figure 2 Evaluation of the objective function changing material (Young's Modulus in MPa) and damage parameters (multiplied by 10)

\section{THE TEST CASE}

The methodology presented in this paper has been applied to a real test case. The case study is a warehouse built in the 1960 when, especially in Italy, the structures where designed just to withstand the vertical loads and not lateral ones given for example by wind or earthquakes. This reinforced concrete structure illustrated from the top view in Figure 3 was composed by two main parts: A) building with the main offices, B) warehouse used for stocking products and production. The structure has been subjected to years of corrosion and chemical materials that contributed to the degradation of the material characteristics.

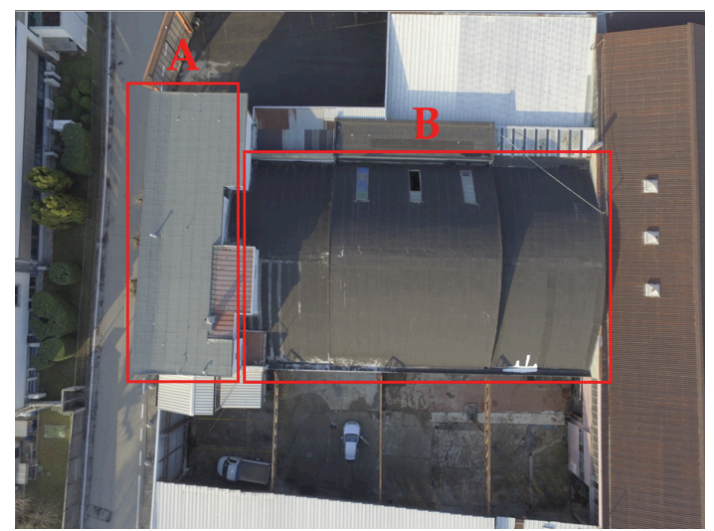

(a)

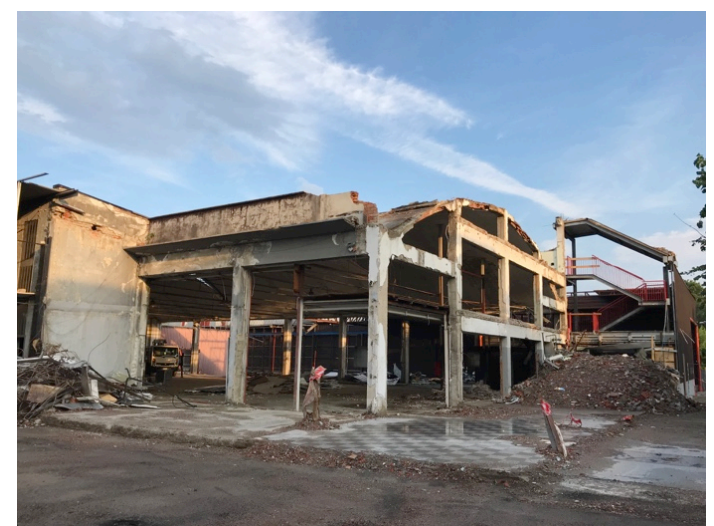

(b)

Figure 3 - Warehouse object of the analysis

Before this experimental campaign, the whole structure has been subjected to significant restoration activities. The building of the main offices has been demolished. In Figure 3 the state of the structure during the analysis and the absence of the building A are shown. 7 bays in the longitudinal direction and 4 bays in the transversal direction compose the warehouse structure. Each bay is $5 \mathrm{~m}$ long and the global dimensions are $35 \mathrm{x} 20 \mathrm{~m}$. The warehouse has 3 main masonry vaults: the first and last sections have a radius of $13.40 \mathrm{~m}$ while the central a radius of $17.90 \mathrm{~m}$. 


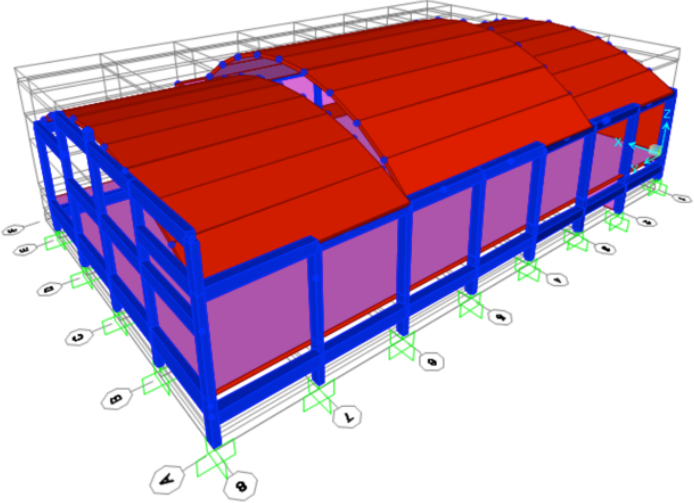

(a)

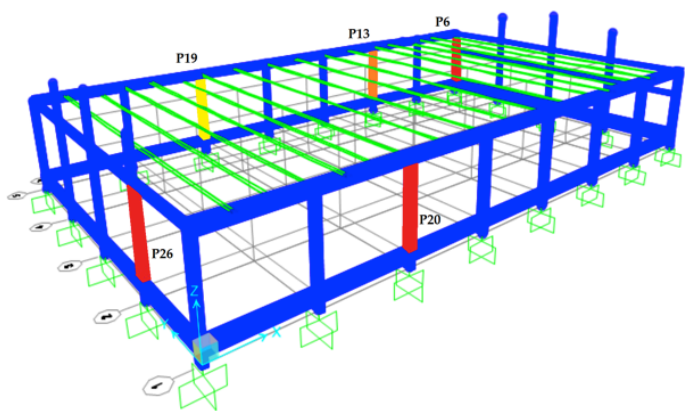

(b)

Figure 4 FEM of the warehouse: (a) complete structure and (b) artificial damages inserted in the model (yellow color [0-30\% damage], orange color [30-70\%], red color [70-100\%])

A FEM of the structure has been realized and it is illustrated in Figure 4a. All the components belonging to the structure have been modeled. In particular, the masonry infill that gives significant contribution to the transversal stiffness of the structure in the linear response field. These have been modelled according to [12] and [13].

Before to elaborate the experimental data, in order to validate the methodology, artificial damages have been inserted in the model, see Figure 4b. In order to demonstrate the capabilities of identifying both material characteristics and local stiffnesses decrease, the identification has been performed on a numerical simulation of the structure excited by white random noise and applying OMA on the data recorded from the simulated damaged model. For the sake of simplicity only an illustration of the damaged configuration is presented.

In Table 2 the name of the columns where the damage has been introduced is shown together with the material adopted. The comparison between the target and the identified values is very good.

\begin{tabular}{ccc}
\hline Name of the element & $\begin{array}{c}\text { Identified damage } \\
\text { parameter }\end{array}$ & $\begin{array}{c}\text { Target damage } \\
\text { parameter }\end{array}$ \\
\hline P6 & 0,75 & 0,75 \\
\hline P13 & 0,50 & 0,50 \\
\hline P19 & 0,20 & 0,20 \\
\hline P20 & 0,80 & 0,80 \\
\hline P26 & 0,70 & 0,70 \\
\hline Material “C25/30" & $31,00 \mathrm{MPa}$ & $31,00 \mathrm{MPa}$ \\
\hline
\end{tabular}

This result has been achieved under some assumptions:

- $\quad$ The modes identified from the simulated damaged structure are the same of the FEM ones;

- $\quad$ Low noise level has been introduced in the identified modal parameters;

- Only some elements of the structure (in this case some columns) have been affected by the damage.

Regarding the last hypothesis, it is worth mentioning that when the update has to deal with incomplete modal data or with structures with particularly extended local damages, the objective function results to be discontinuous because of the MAC criterion, used to compare the mode shapes. Thanks to this strategy extended damages can be identified, but this discontinuity requires global optimization algorithms, such as Genetic Algorithms (GA) or Particle Swarm algorithms (PSO), that exponentially increase the computational effort. 


\section{THE EXPERIMENTAL CAMPAIGN}

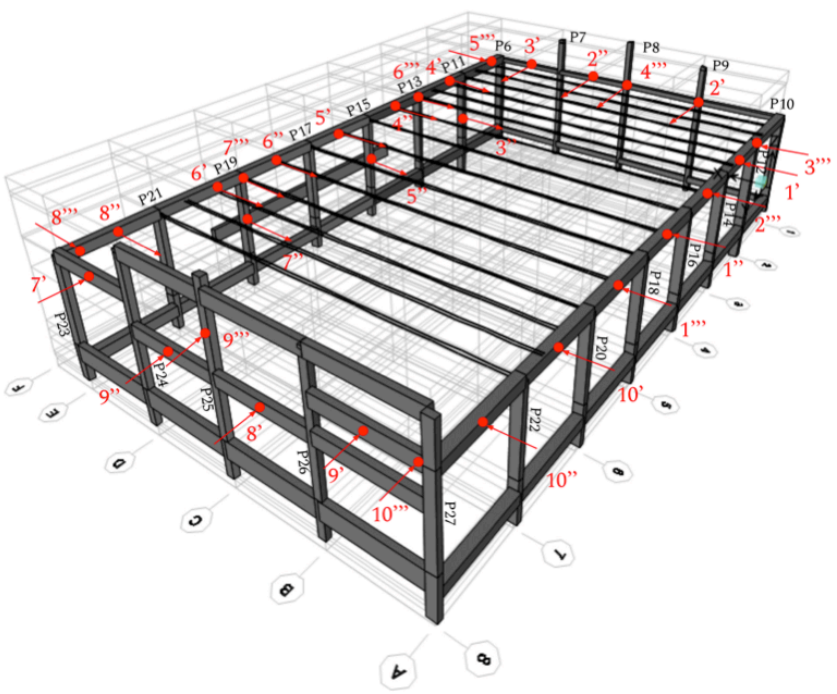

5. Figure 5 Scheme of the measurement locations

Figure 5 shows the configuration of the monoaxial piezoelectric accelerometers $(\mathrm{PCB} 393 \mathrm{~B} 12$, measurement range $=0.5 \mathrm{~g}$ $\mathrm{pk}$, frequency range $=0.05-4000 \mathrm{~Hz}$, sensitivity $=10000 \mathrm{mV} / \mathrm{g}$ ) installed on the structure.

Different time histories have been recorded in 3 moments of the test day with 10 accelerometers (respectively indicated with 3 apex). However, in order to avoid a missing of information, one channel has been kept constant during all the measurements. This allows to reconstruct the mode shapes with 30 points. Figure 6 reports some photos of the sensors installed on the structure.
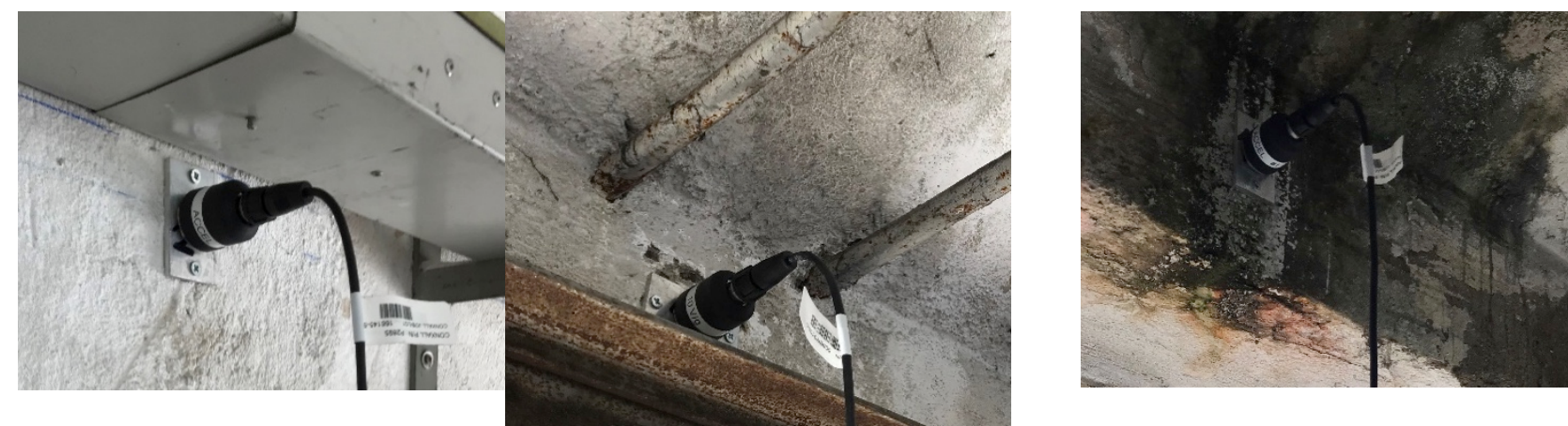

Figure 6 Piezoelectric accelerometers used for the measurements

The accelerometer signals have been recorded at $2000 \mathrm{~Hz}$ and a low pass Butterworth filter has been applied at $1000 \mathrm{~Hz}$. The vibrations of the structure due to ambient excitation, such as wind and traffic, have been recorded for $600 \mathrm{~s}$. In Figure 7(a) and Figure 7(b) it is possible to see respectively: the whole time history recorded during one of the three records and the detail of the acceleration measured by the 10 sensors. 


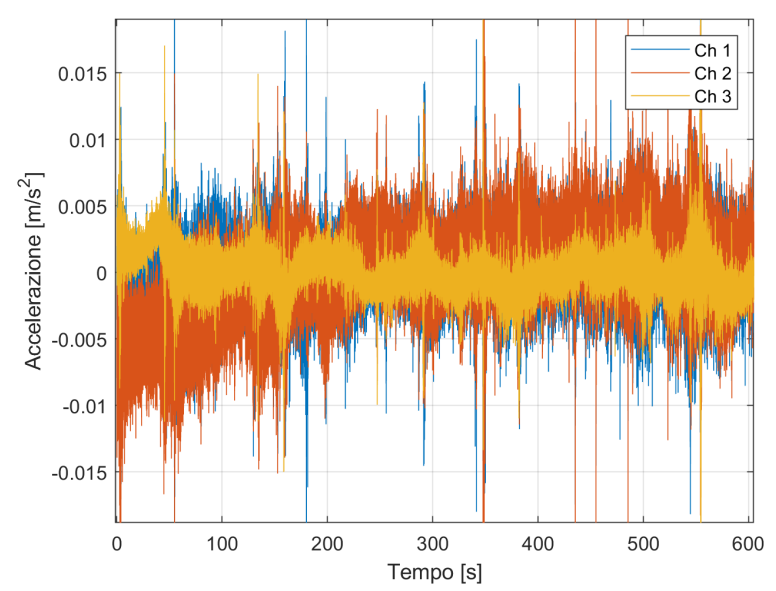

(a)

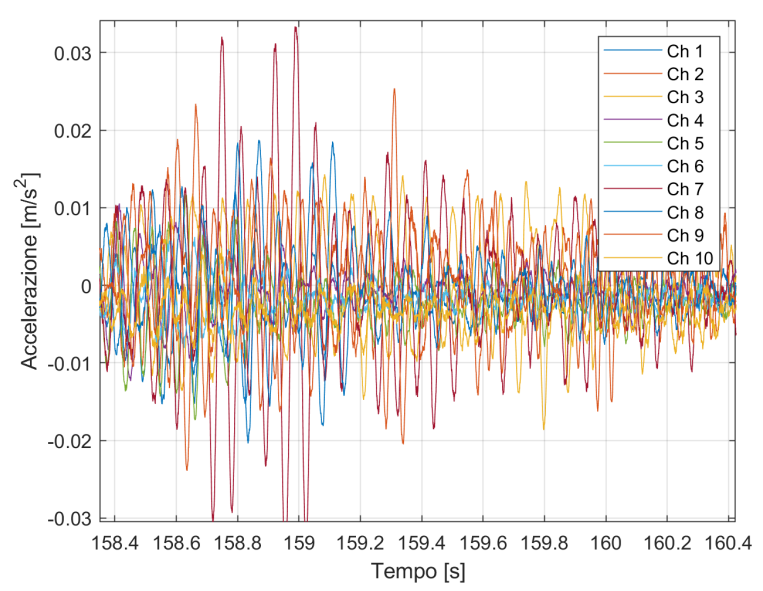

(b)

Figure 7 Measured acceleration of different sensors during the recording of the structure vibrations: (a) whole time history of the first 3 channels of the first record (b) acceleration spike detail of all the measured accelerations recorded by all the sensors

In order to perform the dynamic identification of the structure, the data have been pre-processed by applying a digital low pass filter and downsampling the data. Indeed, as shown in Figure 8 with the Power Spectral Density (PSD) of the recorded data, the main part of the signal power is concentrated in the lower frequency range. For this reason all the following elaborations will be focused in the range below $10 \mathrm{~Hz}$.

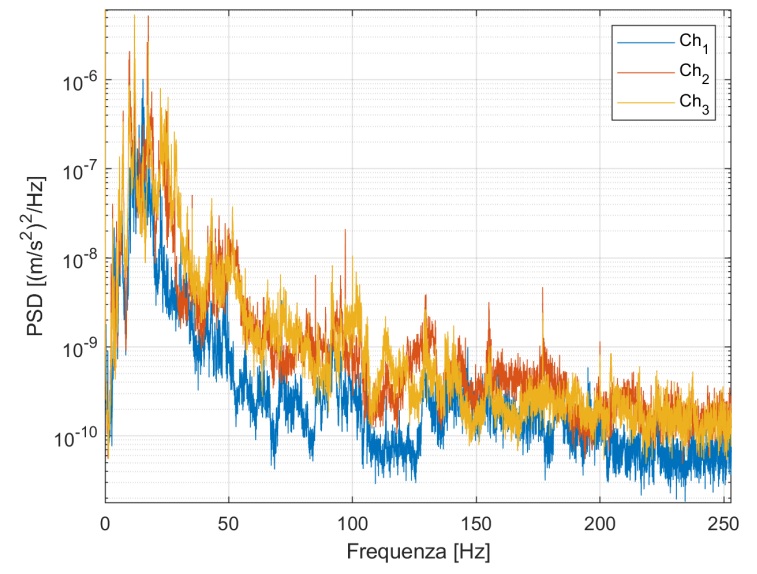

(a)

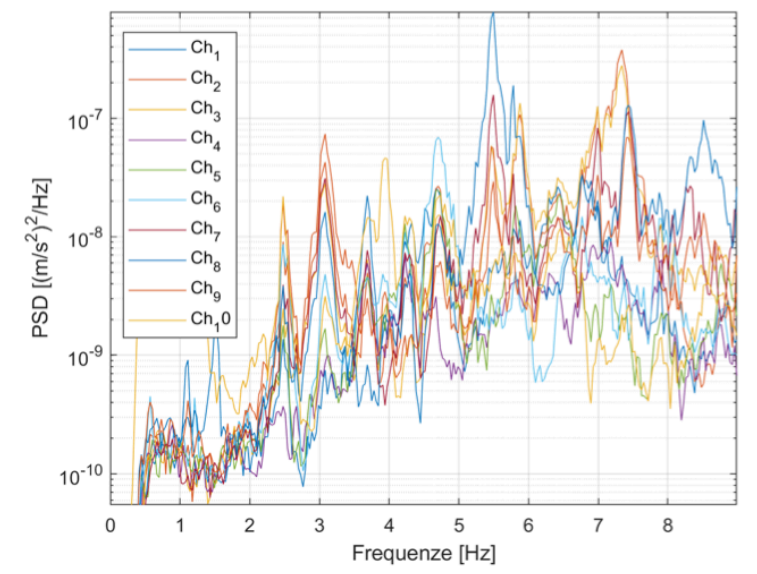

(b)

Figure 8 PSD of the measured accelerometers

Moreover, during the tests, also the bending vibrations of the tendons of the warehouse have been recorded. Each tendon has been excited with an impulse and the free decay of the cable is acquired. Thanks to this strategy, it has been possible to study the fundamental frequency of the cable $(\omega)$, which is directly correlated to the cable tensile load, according to the equation:

$$
T=m \cdot\left(\frac{\omega L}{\pi}\right)^{2}
$$

where $m$, is mass per unit length of the cable $[\mathrm{kg} / \mathrm{m}]$ and $L$ is the total length of the cable $[\mathrm{m}]$. Figure $9(\mathrm{a})$ shows the acceleration measured on one of the tendons. It can be clearly stated that the motion is monoharmonic and from Figure 9 (b) the fundamental frequency is easily computed. 


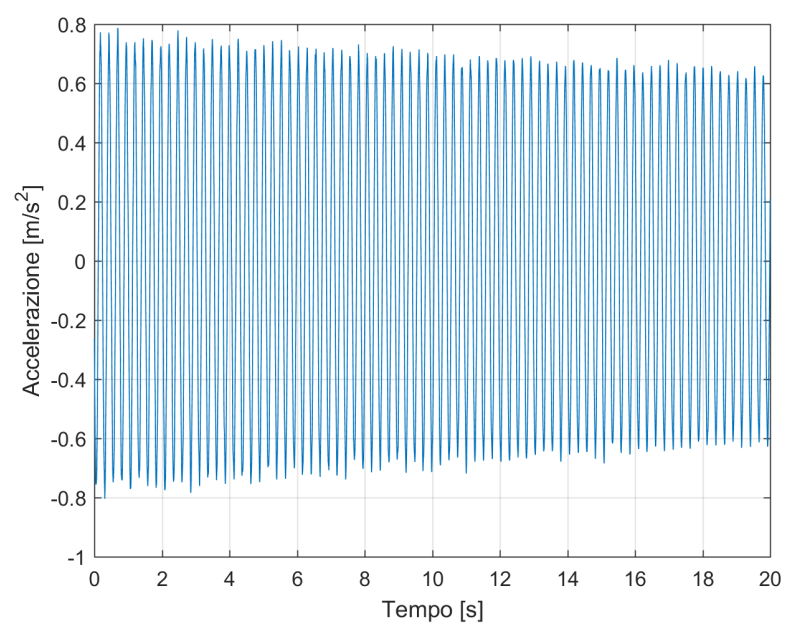

(a)

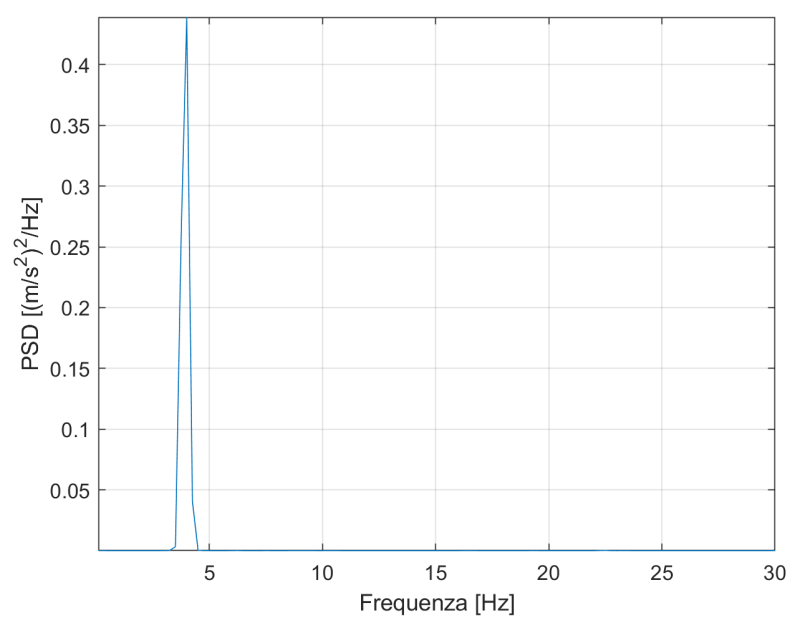

(b)

Figure 9 (a) acceleration of one of the tendons of the structure after impulse excitation (b) PSD of the acceleration signal

Finally, the OMA algorithms have been applied and in Figure 10 the stabilization diagram over the autocorrelation spectrum of the first 3 accelerometers are presented. It is worth to remember that the data have been processed using as reference channel kept constant for the whole campaign.

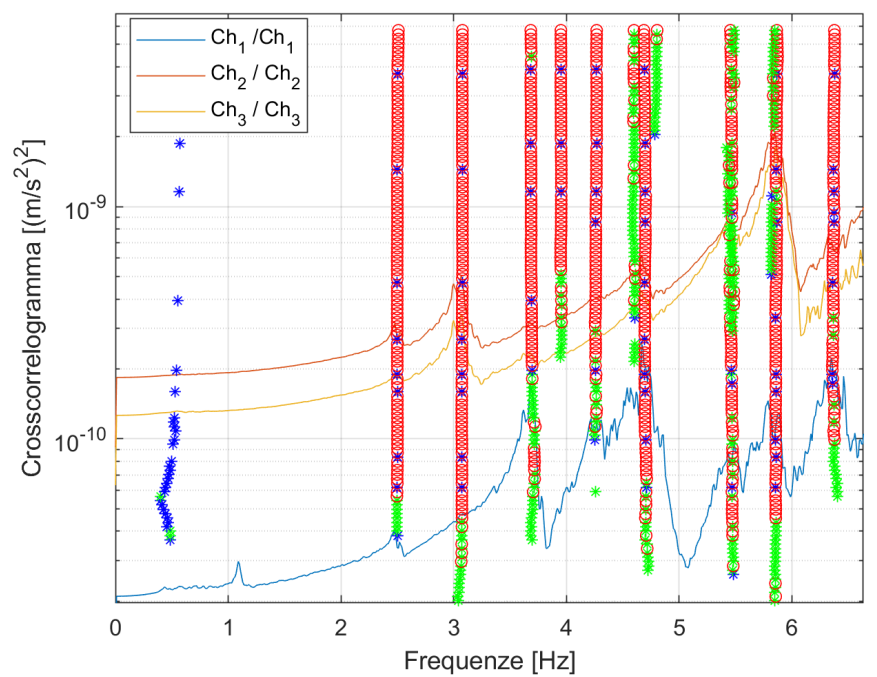

Figure 10 Stabilization diagram obtained from the applied OMA algorithms

The results obtained are listed in Table 3 and, as a proof of the validity of the identification process, in Figure 11 the obtained frequencies have been plotted on the PSD and on the singular value plot obtained through the Singular Value Decomposition (SVD) of the computed Power Spectral Density matrix.

\begin{tabular}{|c|c|c|c|c|c|c|c|c|c|c|}
\hline Mode $n^{\circ}$ & 1 & 2 & 3 & 4 & 5 & 6 & 7 & 8 & 9 & 10 \\
\hline$f_{\text {exp }}[H z]$ & 2,500 & 3,076 & 3,687 & 3,953 & 4,265 & 4,698 & 5,465 & 5,862 & 6,373 & 6,786 \\
\hline$\xi[\%]$ & 1,14 & 2,25 & 0,87 & 0,90 & 1,21 & 1,28 & 0,51 & 0,95 & 1,45 & 0,55 \\
\hline
\end{tabular}

Table 3 Obtained parameters from the dynamic identification of the structure 


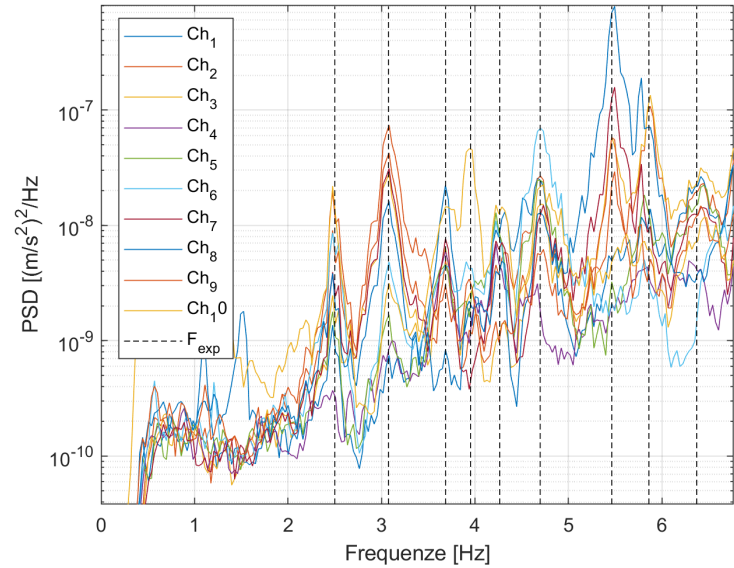

(a)

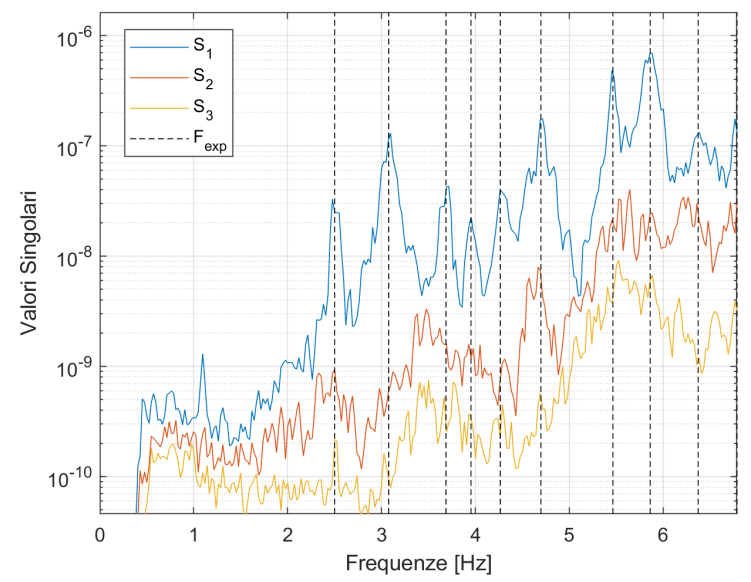

(b)

Figure 11 (a) PSD vs. OMA result; (b) Singuar values plot vs. OMA result

Besides the natural frequencies and damping ratios, also the mode shapes have been computed. Figure 12 shows a top view of the first four mode shapes.

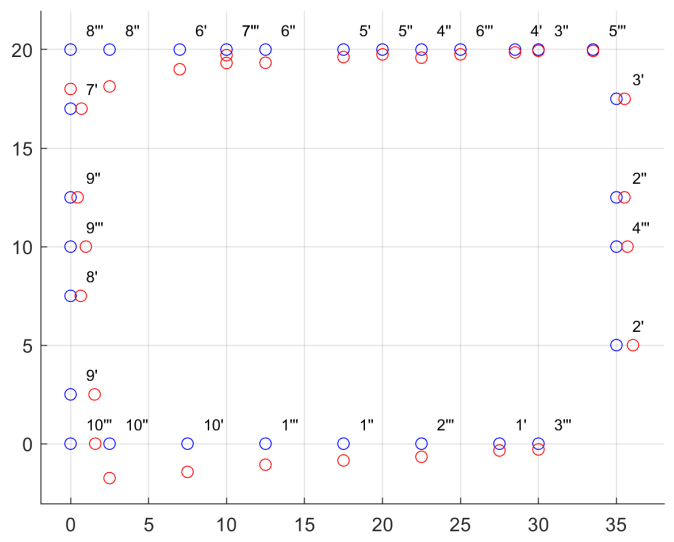

(a)

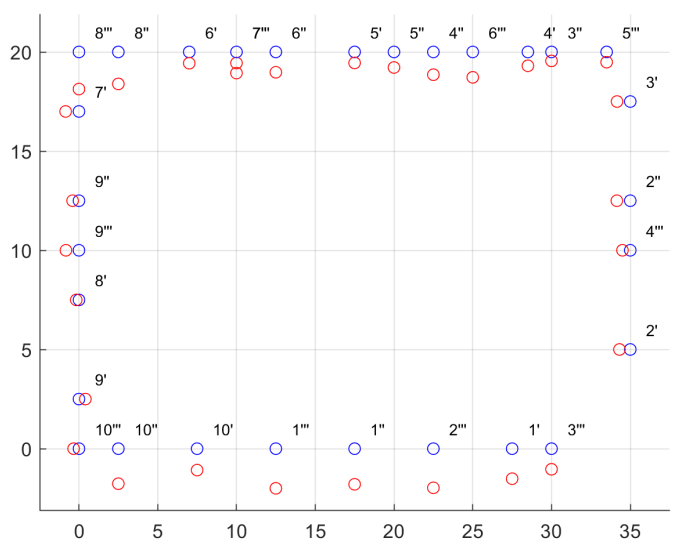

(c)

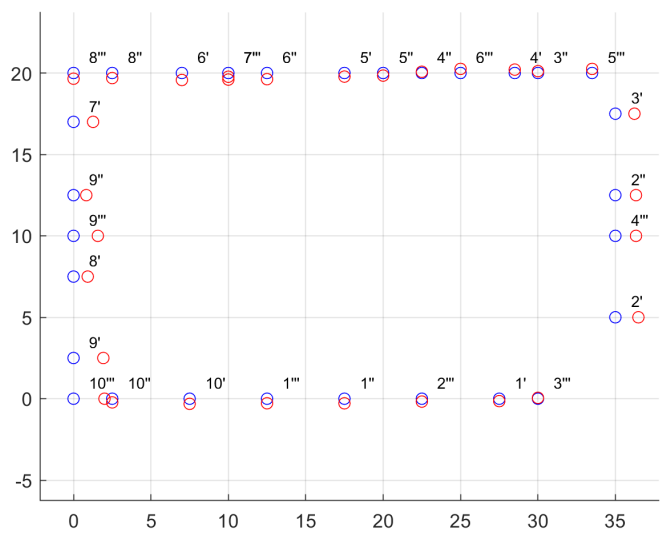

(b)

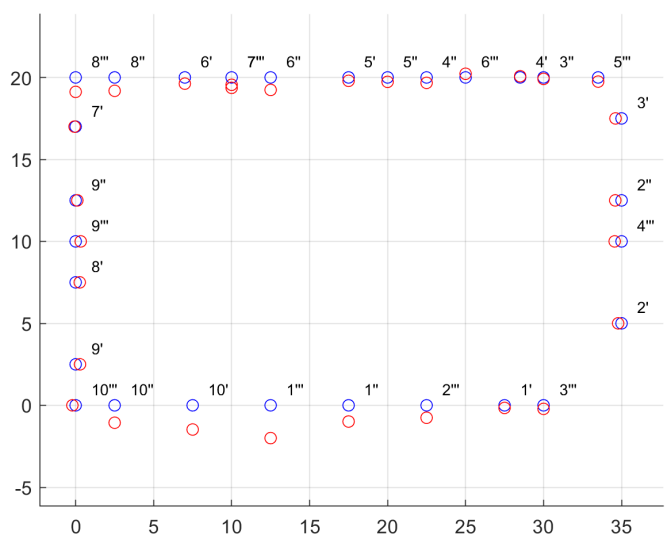

(d)

Figure 12 Obtained mode shapes from the analysis (a) first mode; (b) second mode; (c) third mode; (d) fourth mode; 


\section{THE FEM UPDATE: RESULTS AND DISCUSSION}

Exploiting the real modal data obtained from the analysis illustrated in the previous chapter, the FEM update has been performed.

Table 4 shows the starting point of the analytical frequencies, obtained from the initial FEM, and the experimental ones. For the update, only the first four modes have been used since, as already demonstrated, they allow a good reconstruction of the structure dynamics.

\begin{tabular}{|l|l|l|l|l|} 
Mode $^{\circ}$ & $\mathbf{1}$ & $\mathbf{2}$ & $\mathbf{3}$ & $\mathbf{4}$ \\
\hline FEM modes [Hz] & 2,851 & 3,393 & 3,964 & 4,499 \\
\hline Experiment modes $[\mathrm{Hz}]$ & 2,500 & 3,076 & 3,687 & 3,953 \\
\hline
\end{tabular}

Table 4 Difference between the natural frequencies of the FEM and the dynamic parameters obtained from experimental tests

The optimization process has been carried out, first optimizing the materials and then the damage factors of all the elements (columns and beams), with a different parameter for each bending direction (2-2 and 3-3).

In Table 5 the final result of the optimization is shown. The frequencies of the updated FEM are almost equal to the experimental ones. Moreover, Table 6 reports the 3D deformed shape of the building coming from the FEM (on the right) and the difference in top view between the experimental identified mode shapes and the analytical ones (on the left). Also in this case a good level of agreement is achieved.

\begin{tabular}{|l|l|l|l|l} 
Mode $^{\circ}$ & $\mathbf{1}$ & $\mathbf{2}$ & $\mathbf{3}$ & $\mathbf{4}$ \\
\hline FEM modes [Hz] & 2,515 & 3,090 & 3,564 & 3,922 \\
\hline Experiment modes $[\mathrm{Hz}]$ & 2,500 & 3,076 & 3,687 & 3,953 \\
\hline
\end{tabular}

Table 5 Difference between the natural frequencies of the FEM and the dynamic parameters of the real structure after the FEM update procedure

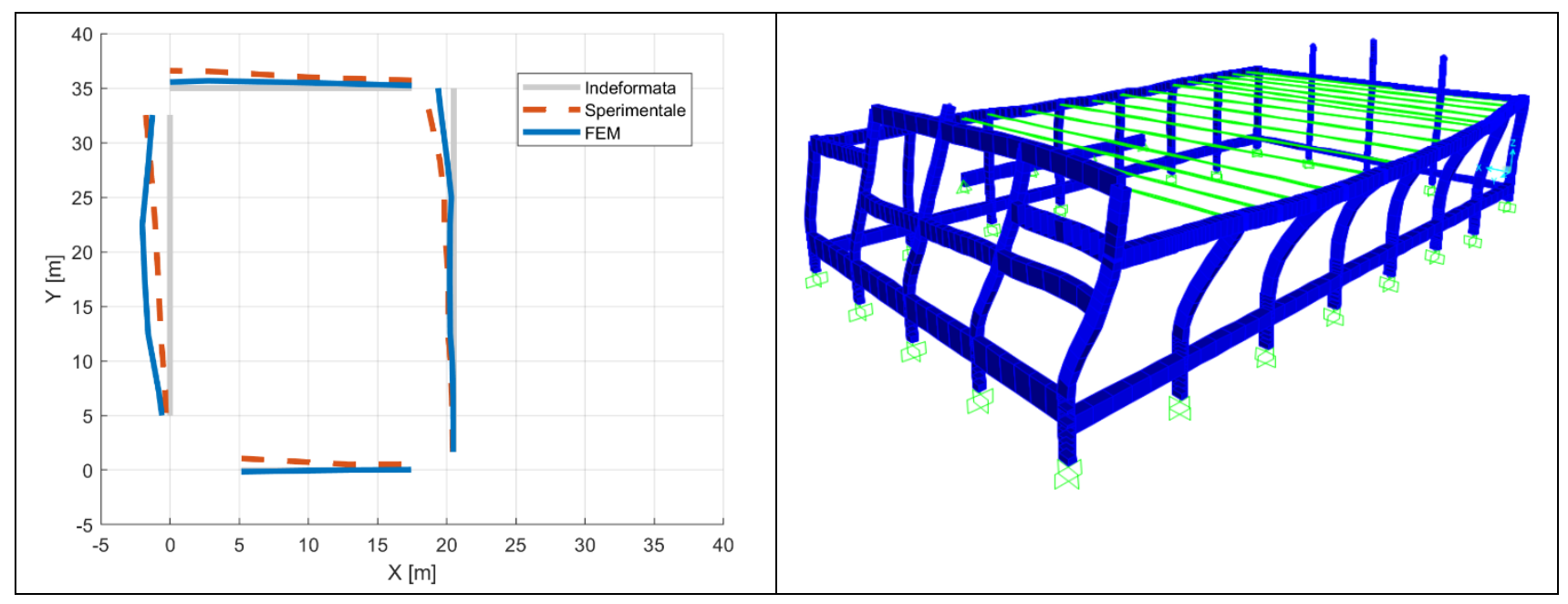




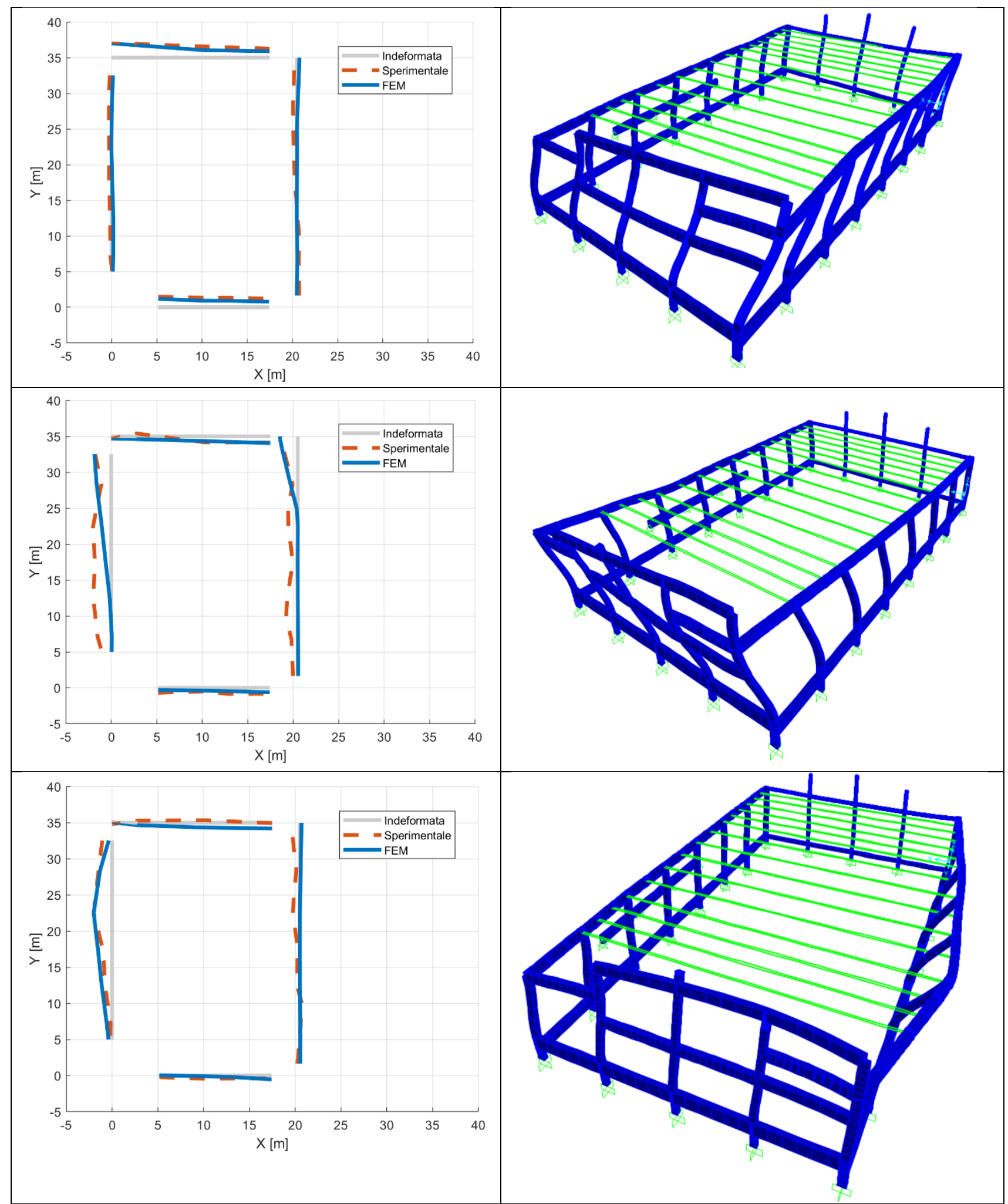

Table 6 Experimental and analytical mode shapes obtained after the optimization procedure

Table 7 reports the results in terms of material mechanical properties before and after the optimization while Table 8 shows a very interesting result regarding the damage parameters identified. 


\begin{tabular}{|l|c|c|c|}
\hline Parameter & Starting value & Final value & Percentage variation \\
\hline Vault material density & $1223\left[\mathrm{~kg} / \mathrm{m}^{3}\right]$ & $1681\left[\mathrm{~kg} / \mathrm{m}^{3}\right]$ & $37,4 \%$ \\
\hline Vault elastic modulus & $12,5[\mathrm{MPa}]$ & $14,6[\mathrm{MPa}]$ & $16,8 \%$ \\
\hline Masonry infill density & $1019\left[\mathrm{~kg} / \mathrm{m}^{3}\right]$ & $1393\left[\mathrm{~kg} / \mathrm{m}^{3}\right]$ & $36,7 \%$ \\
\hline Masonry infill elastic modulus & $12,5[\mathrm{MPa}]$ & $10,2[\mathrm{MPa}]$ & $18,4 \%$ \\
\hline Concrete density & $2584\left[\mathrm{~kg} / \mathrm{m}^{3}\right]$ & $2582\left[\mathrm{~kg} / \mathrm{m}^{3}\right]$ & $0,08 \%$ \\
\hline Concrete elastic modulus & $33,3[\mathrm{MPa}]$ & $34,4[\mathrm{MPa}]$ & $3,03 \%$ \\
\hline
\end{tabular}

Table 7 Results of the parameter mechanical properties obtained after the FEM update

\begin{tabular}{l|l|l|l|l|l|l|l|l|l|l|l|l|l|l|l|l|} 
Beams names & T1 & T3 & T4 & T7 & T8 & T1 & T1 & T1 & T1 & T1 & T2 & T2 & T2 & T2 & T2 \\
\hline & & & & & $\mathbf{0}$ & $\mathbf{2}$ & $\mathbf{5}$ & $\mathbf{6}$ & $\mathbf{7}$ & $\mathbf{0}$ & $\mathbf{2}$ & $\mathbf{4}$ & $\mathbf{5}$ & $\mathbf{6}$ \\
\hline I 2-2 Damage [\%] & $/$ & $/$ & 20 & 30 & 70 & $/$ & 40 & $/$ & $/$ & 50 & 50 & $/$ & $/$ & $/$ & 50 \\
\hline I 3-3 Damage [\%] & 50 & 40 & $/$ & $/$ & $/$ & 40 & 20 & 20 & 30 & $/$ & $/$ & 30 & 40 & 50 & $/$ \\
\hline
\end{tabular}

Some important remarks have to be underlined about these results. The main uncertainty in the structure where the material mechanical properties of the masonry infill and the vault, while the columns concrete has been analyzed both with destructive and non-destructive tests that gave as a result the first input values used in the update. Indeed, it is possible to notice that the material mechanical properties didn't change significantly if compared with the initial guess of the other values.

Moreover, for what concerns the obtained damage parameters, these have been checked through a visual inspection of the structure. Some of the elements that resulted to have a high level of damage showed a high degree of corrosion and surface degradation, as shown for instance in Figure 13 (elements T1 and T8).

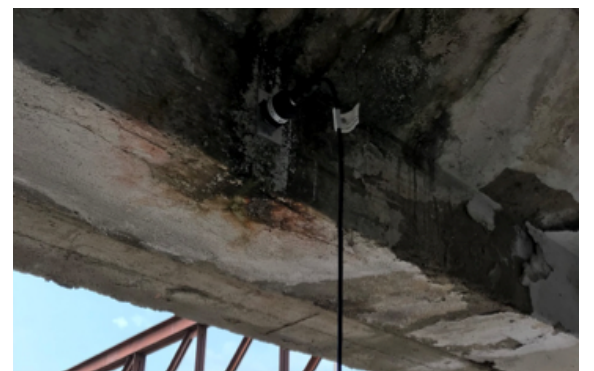

(a)

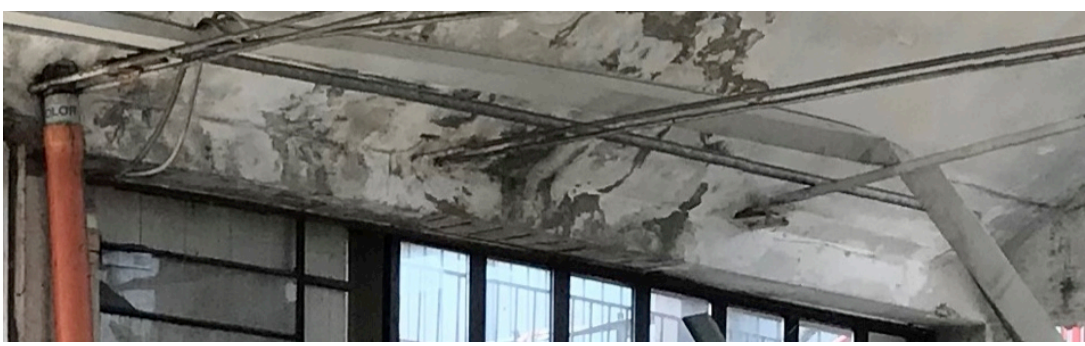

(b)

Figure 13 (a) beam T8; (b) beam T1

Also other beams, such as T4 and T15, which didn't highlight issues during the visual inspection (see Figure 14), after a deeper analysis presented an important honey-comb effect probably due to a poor quality of the concrete pouring at the construction phase.

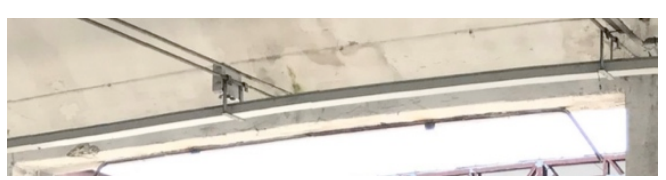

(a)

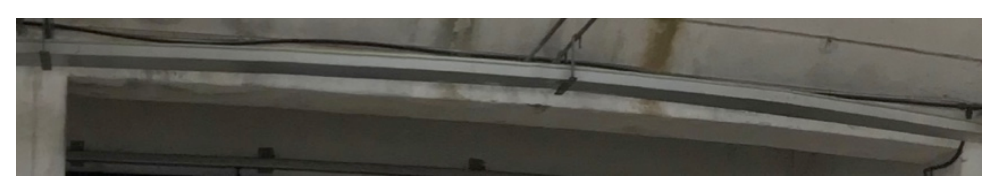

(b)

Figure 14 (a) beam T4; (b) beam T15

\section{CONCLUSIONS}

This work presented an application of the FEM update technique using modal parameters obtained from the OMA. The analysis showed promising results, both for the update of the material mechanical properties and for the damage identification, supporting the engineering during the structure diagnostics. The results have been briefly discussed and some important conclusions can be drawn with respect to computational effort and the degree of uncertainty. 
The degree of uncertainty in the results is strictly related to the inverse problem. This could be mitigated through a good model of the structure and by using starting parameters close to the real ones. For these reasons, some local tests have to be still performed, although less expensive in terms of both time and costs.

Moreover, due to the complexity of the objective function, global optimization algorithms, such as Genetic Algorithms or Particol Swarm algorithms, need to be employed, increasing the computational time and lowering the maximum number of parameters that can be updated.

In conclusion, this technique could strongly change the approach to the civil structural analysis, allowing to obtain more reliable FEM and standardizing a procedure, that nowadays still shows a too high variability of methods.

\section{AKNOWLEGMENTS}

This study is part of an ongoing research collaboration between ISAAC s.r.l. and Politecnico di Milano. The authors wish to thank the ISAAC s.r.l. members for their continuous support and valuable contribution to the success of this research project.

\section{REFERENCES}

[1] G. De Roeck, E. Reynders, P. G. Bakir, “An improved finite element model updating method by the global optimization technique 'Coupled Local Minimizers"”, Computer and Structures 2007

[2] D.Foti, M. Diaferio, N. I. Giannoccaro, M. Mongelli, "Ambient vibration testing, dynamic identification and model updating of a historic tower", NDT\&E International 2011

[3] D.Skolnik, Y. Lei, E. Yu, J. W. Wallace "Identification, Model Updating, and Response Prediction of an instrumented 15-story steel frame building”, Earthquake Spectra 2006

[4] Ripamonti, F., Chiarabaglio, A. A smart solution for improving ride comfort in high-speed railway vehicles (2019) JVC/Journal of Vibration and Control, 25 (13), pp. 1958-1973.

[5] Ripamonti, F., Cola, F. Control system for a carbon fiber plate using an adaptive negative derivative feedback control algorithm (2018) JVC/Journal of Vibration and Control, 24 (21), pp. 4988-4999.

[6] Ripamonti, F., Orsini, L., Resta, F. A Nonlinear Sliding Surface in Sliding Mode Control to Reduce Vibrations of a Three-Link Flexible Manipulator (2017) Journal of Vibration and Acoustics, Transactions of the ASME, 139 (5), art. no. 051005, .

[7] Serra, M., Resta, F., Ripamonti, F. Dependent modal space control: Experimental test rig (2017) JVC/Journal of Vibration and Control, 23 (15), pp. 2418-2429.

[8] Ripamonti, F., Leo, E., Resta, F. Experimental and numerical comparison between two nonlinear control logics (2016) International Journal of Applied Mechanics, 8 (5), art. no. 1650061, .

[9] Cinquemani, S., Diana, G., Fossati, L., Ripamonti, F. A smart structure for wind tunnel investigation of a bridge deck's vortex-induced torsional motion (2016) Mechatronics, 33, pp. 108-120.

[10] J. E. Mottershead, M. Link, M, I, Friswell "The sensitivity method in finite element model updating: A tutorial”, Mechanical Systems and Signal Processing 2010

[11] M. Aghagholizadeh, F.N. Catbas "A review of model updating methods for civil infrastructure systems", Computational techniques for Civil and Structural Engineering 2015

[12] T. M. Alguhane, A. H. Khalil, M. N. Fayed, A. M. Ismail, "Modeling of Masonry In-Filled R/C Frame to Evaluate Seismic Performance of Existing Building”, International Journal of Civil and Environmental Engineering 2015

[13] L. Cavàleri, M. Papia, G. Macaluso, F. Di Trapani, P. Colajanni, “Definition of diagonal Poisson's ratio and elastic modulus for infill masonry walls", Materials and Structures 2012 